AperTO - Archivio Istituzionale Open Access dell'Università di Torino

Rehabilitative intervention during and after pediatric hematopoietic stem cell transplantation: An analysis of the existing literature

This is a pre print version of the following article:

Original Citation:

Availability:

This version is available http://hdl.handle.net/2318/1690350

since 2020-04-14T12:19:45Z

Published version:

DOI:10.1002/pbc.26114

Terms of use:

Open Access

Anyone can freely access the full text of works made available as "Open Access". Works made available under a Creative Commons license can be used according to the terms and conditions of said license. Use of all other works requires consent of the right holder (author or publisher) if not exempted from copyright protection by the applicable law. 


\section{Title page}

Title: Rehabilitative intervention during and after pediatric hematopoietic stem cells transplantation: an analysis of the existing literature.

Running title: Rehabilitation in stem cells transplantation.

Authors: Francesca Rossi ${ }^{* 1}$, Monica Coppo*2, Giulia Zucchetti ${ }^{3}$, Franca Fagioli ${ }^{3}$.

1 Rehabilitation Service, Pediatric Neurology and Psychiatry, A.O.U. Città della Salute e della Scienza - Ospedale Infantile "Regina Margherita", Turin, Italy

2 Degree Course of Neuro-Psychomotor Therapy of Developmental Age, Medicine University of Turin, Italy.

3 Pediatric Oncohematology, Stem Cell Transplantation and Cell Therapy Division. A.O.U. Città della Salute e della Scienza - Ospedale Infantile "Regina Margherita”, Turin, Italy

Address correspondence to Francesca Rossi, Rehabilitation Service, Pediatric Neurology and Psychiatry, A.O.U. Città della Salute e della Scienza - Ospedale Infantile "Regina Margherita”, Turin, Italy, Piazza Polonia 94, 10126, Turin, Italy.

Telephone: 0113131643

E-mail: francesca.rossi@unito.it

All authors declare no conflict of interest about the following manuscript. 


\title{
Rehabilitative intervention during and after pediatric hematopoietic stem cells transplantation: an analysis of the existing literature.
}

\begin{abstract}
$\underline{\text { Abstract }}$
Hematopoietic Stem Cell Transplantation (HSCT) is a therapeutic strategy for several oncohaematological diseases. It greatly increases long-term survival rates but growths the incidence of related-effects on physical, neuropsychological and functional functioning with negative impact on of quality of life (QoL).

The objective of this paper was to summarize and compare the existing literature focused on physical exercise interventions among pediatric HSCT recipients to explore the most employed rehabilitative assessment and treatment tools.

Relevant studies published up to April 1, 2015 were selected from PudMed, The Cochrane Library, CINAHL, Scopus, PEDro and PsycINFO: 10 studies with different research types were included. Proposed rehabilitative interventions was performed before, during, and after infusion. In this paper, we subdivided articles on the base of moment of intervention in the course of transplant process (during hospitalization and after discharge) dedicating a paragraph to studies focused on management of cGVHD.

A previous literary review has already shown that rehabilitation programs improve physical fitness and functional abilities with a positive influence on (QoL). Thanks to our analysis, we could identify some significant outcome variables and shared intervention areas suitable for clinical practice and trials design. In fact, further studies are required for validation of rehabilitative guidelines.
\end{abstract}




\section{$\underline{\text { Introduction }}$}

Hematopoietic stem cell transplantation (HSCT) involves intravenous infusion of autologous or allogeneic stem cells collected from bone marrow, peripheral blood, or umbilical cord blood preceded by high-dose chemotherapy and, if appropriate total-body irradiation, to re-establish hematopoietic function in patients whose immune system is damaged or defective(1). HSCT is a potential curative option for many diseases, such as hematologic malignancies and refractory solid tumors. This procedure led to considerable improvement in survival rates, but also determined the onset of late effects-related problems(2-10). Due to the severity of conditioning regimen, length of isolation and bed rest period, immunosuppression and drug toxicities, it seriously impairs children's physical functioning. HSCT recipients may suffer impairments in cardiorespiratory fitness (with high incidence of severe fatigue), muscle trophysm and strength, immunologic recovery (with high risk of infections), functional abilities and autonomy(3-5,11). These elements determine an undeniable decline of quality of life (QoL) in pediatric cancer survivors(12-15). In addition, graft versus host disease (GVHD) is a frequent complication of allo-HSCT(16). The incidence of the chronic form (cGVHD) has been estimated between $30-70 \%$ in those surviving beyond 100 days, with an average onset of 4-6 months after transplant. cGVHD symptoms, such as gradual loss of lung function, loss of flexibility and strength, very weak state of health, articular angle reduction, low functional abilities, cause a vicious circle that induces immobility with a consequent deterioration of physical functioning(17-21).

Some studies have already shown the feasibility, safety and effectiveness of a rehabilitative program during and after HSCT's period (22-27) but they very heterogeneous in terms of kind of study, outcome measures, rehabilitative assessments tools and treatment programs. A recent review, conducted by West et al., 2014(27), focused on impact of exercise programs on physical activity in this population, concluded that exercise as medicine in a non-invasive and non-pharmaceutical treatment that may be used in management of physical morbidity but exercise prescription guidelines are needed. According to these conclusions, we conducted a literature analysis focusing on intervention programs and proposed instruments for rehabilitative assessment and treatment. The aim was to synthesize knowledge and author operating tips diversifying them by the moment of intervention: during hospitalization (in-patients intervention) and after discharge (out-patients 
intervention). We hope to establish expendable guidelines for clinical practice and for further studies about physical exercise among children and adolescents treated with HSCT.

\section{$\underline{\text { Methods }}$}

This paper included studies on rehabilitative assessment and treatment of children undergoing HSCT for the treatment of different onco-haematological diseases. Studies were chosen considering as inclusion criteria: published until April 1, 2015; involving patients aged 0-18 years; focused on rehabilitative assessment and/or treatment of HSCT effects; published in English.

Relevant studies were identified conducting research on 6 biomedical databases: PubMed, The Cochrane Library, CINAHL, Scopus, PsycInfo and PEDro. In order to compose the research array, the primary search terms "Hematopoietic stem cell transplantation”, " Pediatric Hematopoietic stem cell transplantation", "Graft versus host disease", were combined with the following key words "Outcomes", "Late effects", "Physical assessment", "Functional assessment", Exercise”, "Rehabilitation", "Motor rehabilitation", "Physical activity" (Mesh terms or free-text words). Some example of full search strategy were: "Hematopoietic stem cell transplantation AND Exercise (Limit: all child)", "Graft versus host disease AND rehabilitation (Limit: all child)". No mesh terms have been used: they seem to be not useful if the argument of research has not a wide literature.

We obtained a recent review(26) involving adults HSCT recipients and took this review as an example for conducting the present research.

Ten studies met the inclusion criteria and were further subdivided into articles focus on: in-patients intervention (beginning at child hospitalization for conditioning therapy and continuing until discharge); out-patients intervention (beginning at discharge and continuing until the end of the first year after transplant); assessment and treatment of Chronic Graft versus Host Disease. 


\section{$\underline{\text { Selected studies analysis }}$}

\section{In-patients phase: rehabilitative assessment and treatment.}

Currently, scientific literature presents 3 studies on exercise programs during hospitalization: Chamorro-Vina et al., 2012(28), Rosenhagen et al.,2011(25), Chamorro-Vina et al., 2010(24). They are a randomized clinical trial (RCT), a controlled clinical trial and a case-control study.

Chamorro-Vina et al., 2012 had the objective of evaluating effects of an exercise program during both in-patients and out-patients phases on immune recovery and on prevention of QoL and physical fitness decline. The sample included 24 patients (5-18 years) undergoing auto-HSCT.

Outcome variables were measured at baseline, +30 days from transplant, $+85,+180$ days. These were: quantitative and functional immune recovery; body mass index (BMI); nutrition; cardiovascular functioning; QoL; fatigue; physical activity levels; motor skills; muscle resistance; strength; muscle elasticity.

Exercise intervention during the in-patient phase (30 days) included aerobic activities and resistance training, consisting in muscle strengthen exercises involving major muscle groups. The out-patients phase intervention will be exposed in the next session of the paper.

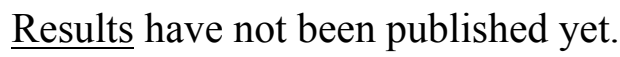

Rosenhagen at al., 2011 had the purpose of assessing feasibility and patient acceptance of supportive physical therapy into isolation phase of PSCT. Their sample included 23 patients $(15.3+/-3.7$ years) undergoing auto/allo-PBSCT. 13 of 23 patients were assigned to intervention group, 10 to the control group.

Outcomes variables were evaluated at recruitment moment and after discharge. These were: motor skills, health-related quality of life (HRQOL); fatigue; acceptance of training intervention. Intervention program involved the in-patients phase (average of 34 days). Sessions were divided in: endurance training (10 minutes) using a stationary bicycle ergometer, strength training, including exercises with balls, and coordination exercises.

Results of the study demonstrate the feasibility of an exercise intervention during in-patients phase. Acceptance for proposed program was high, too. Authors showed that ergometer training lead to stabilization or even a slight improvement of physical performance with a higher $\mathrm{VO}_{2}$ max and a lower 
resting heart rate. Moreover, decline of manual force could be prevented. Implemented physical activity also leads to progress in QoL and cancer-related fatigue; ultimately, it helps children to trust in their physical abilities, enabling them to regain movement skills earlier in the phase of rehabilitation and keeps strength and endurance during the isolation phase on a functional level.

Chamorro-Vina et al., 2010 had the objective of evaluating the effects of a 3 weeks-rehabilitative intervention performed during inpatient stay for HSCT on immune cell recovery and on physical fitness. Their sample included 7 patients (4-16 years) undergoing allo-HSCT. The control group included 13 patients $(7+/-3$ years $)$ following the same transplant protocol. Outcome variables measured before, 15 and 30 days after HSCT, were: immune recovery, BMI and fat-free mass (FFM). Intervention program started at the beginning of conditioning phase and lasted until the end of neutropenic phase (about 3 weeks). The treatment was subdivided in 5 session with the following organization: articular motion and stretching exercise at the beginning/end of each session; aerobic training with low to moderate cycle ergometer (25-30 mins) and strength exercise engaging the majority muscle groups performing on set of 12-15 repetitions.

The results of the study showed that exercise interventions in in-patients did not negatively affect immune cell recovery. Moreover, referred effects on anthropometric variables contributed to increase the patient's body mass, BMI and fat-free-mass. Training program improved children's functional capacity, that increased over intervention period.

\section{Out-patients phase: rehabilitative assessment and treatment.}

Three studies refer to post-transplant phase: Chamorro-Vina et al.,2012(28), San Juan et al., $\underline{\mathbf{2 0 0 8}}(29)$, Hayes et al.,2004(30). The first is a RCT, the other are clinical trials.

First part of Chamorro-Vina et al., 2012 was already exposed in the previous paragraph. The outpatients intervention (10 weeks after discharge) consisted of 2 unsupervised weekly/sessions and 1 supervised home-based training. Supervised session was similar to in-patient intervention consisting of combined aerobic, resistance and strength training; the last involved the major muscle groups with 12-15 repetitions for each exercise set. Home-based program included 20-30 minutes of aerobic 
exercises and 30 minutes of strength and stretching exercise using Nintendo Wii devices. Training adherence were reported in a logbook. Results have not been published yet.

The purpose of San Juan et al., 2008 was to determine if eight-week intrahospital supervised program improves functional capacity and QoL in children HSCT recipients. Sample included 8 patients (8-16 years) who received haploidentical or allo-HSCT in the last 2-12 months; 8 healthy children were selected as a group control.

Outcome variables, evaluated before and after intervention, were: $\mathrm{VO}_{2 \text { peak }}$ and ventilator threshold; gas-exchange-data; strength; resistance; functional mobility; passive and active ankle dorsiflexion; QoL.

Exercise program (8 weeks) begun in a range of 2-12 months after transplant. Sessions started and ended with a low intensity 15-min warm up and cool-down period consisting of cycle ergometer 7edaling and stretching exercises (8-15 repetitions). Training was divided into aerobic and strength exercises. The first consisted of 7edaling on a cycle ergometer, running, walking and aerobic games (group activities, exercise with ball...). Patients started with $10 \mathrm{~min}$ of aerobic exercises at 50\% agepredicted maximum heart rate and progressed to 30 min of continuous exercise at $\geq 70 \% \mathrm{HR}_{\max }$. Stregth training included 11 exercises engaging the major muscle groups on set of 8-15 repetitions.

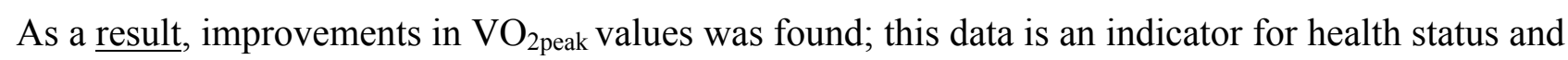
predictor of mortality. Significant training-induced improvements have been found in tests on functional abilities. Authors obtained enhancement in children's and parent's self-reported QoL, too. They underlined the need of performing individually supervised training programs instead of homebased training and the importance of including both aerobic and resistance exercises.

Hayes et.al, 2004 had the aim of evaluating the impact of HSCT on QoL and determining the effect of a mixed type, moderate intensity exercise program on QoL. The sample included 12 patients (1664 years) undergoing auto-HSCT. During the first and the second phase all patients were considered to be in the same group; in the third phase patients were allocated to the control-stretching group and to the intervention group.

Outcome variables, evaluated 7-10 days before admission for the high dose chemotherapy regimen, the day after infusion and after 3 months was : QoL and peak aerobic capacity. 
Intervention group followed a rehabilitation program (12 weeks) consisting of aerobic and resistance exercises. Aerobic component involved a combination of treadmill, walking and stationary cycling (3 times per week, for 20-40 minutes). Intensity and duration of exercises gradually increased. Machine weights used for resistance-training was set to induce failure between 15 and 20 repetitions during weeks 1-6, followed by 8-12 repetitions during 7-12 weeks. All patients kept physical activity logbooks.

As a result, this study demonstrated statistically and clinically significant improvements in all measures of QoL. Moreover, peak aerobic capacity seems to be associated with global and physical functioning and QoL: higher levels of fitness cause higher QoL.

\section{Assessment and treatment of cGVHD.}

Selected articles regarding cGVHD were 4: 2 of them investigates the role of rehabilitative intervention: Carenzio et al., 2007(18) and Currie et al., 2002(19). They are both case-reports. The other were observational studies concern the validation of motor abilities assessment tools in pediatric patients affected by cGVHD: Pidala et al., 2013 (31) and L. Li et al, 2008(32).

Carenzio et al., 2007 was aimed at evaluating clinical and functional assessment and results obtained following rehabilitative treatment in children affected by cGVHD. It presents 6 case-reports of patients (8-17 years) affected by HSCT-related cGVHD. Clinical and functional assessment, performed prior to rehabilitative treatment, after 6 and 12 months, included these outcome variables: range of movement (ROM), extension of skin graft, localization of ulcers, functional abilities and spirometry.

The rehabilitative intervention (1 year)involved: stretching and weight-bearing exercises; exercises to improve change of body position and postural control; massotherapy; occupational therapy; stroking and friction massage for skin adhesions and skin stretching; chest therapy for increase thorax expansion and bronchial clearance.

$\underline{\text { Results }}$ refer that physical conditions improved in 3 cases, remained stationary in 2 cases and worsened in 1 case. Patients showed improvements in functional abilities; rehabilitation favored school return and socialization and the collaboration of child's parents appeared fundamental in home-training. Authors concluded that cGVHD needs rehabilitation at onset of first cutaneous signs. 
Currie et al, 2002 had the aim to report results of a topical treatment of sclerodermoid cGVHD and comment on the rationale of its treatment program. Authors explain the case-report of a 14 -year-old

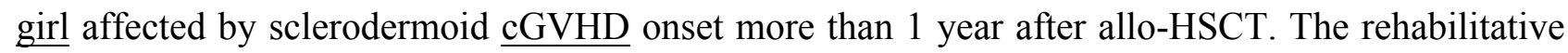
assessment, continuously performed during intervention, included as outcome variables: ROM (arms and legs), evaluation of skin condition and assessment of functional abilities. The rehabilitative treatment consisted of: topical treatment of skin lesions with application of paraffin baths at a temperature of $48^{\circ}$ at the beginning of each session; physical therapy (mobilization, stretching) for improving ROM; strength training; occupational therapy; parent's training of exercise program and use of paraffin. As a result, treatment brought improvements in ROM, skin condition and functional abilities. Authors underlined the importance of physical and occupational therapy with paraffins bath early in the treatment so that more severe impairments could be prevented.

Pidala et al., 2013 had the objective of examining recommended functional measures, 2 Minute Walk Test (2MWT) and Hand Grip Strength Test (HGS), among cGVHD-affected HSCT recipients to determine their association with patient-reported measures, cGVHD severity, response and mortality. The sample of the study involved 584 patients (2-79 years) who undergone allo-HSCT. They were divided into incidence (enrolment $<3$ months after cGVHD diagnosis) and prevalent (enrolment $\geq 3$ months after cGVHD diagnosis but $<3$ years after HSCT) cases. Only 14 patients were children. Outcome measures included: cGVDH symptoms; energy expenditure and physical fitness; QoL; physical functioning.

In the assessment of HGS, strength was measured 3 times from the dominant hand using portable electronic dynamometer. In the conduct of the $2 \mathrm{MWT}$ the patient walked 50-foot course, and the total distance covered in 2 minutes was recorded.

Results showed an independent association of the 2MWT with mortality of cGVHD affected patients. Impaired 2MWT was significantly associated with higher symptoms burden, impaired QoL, functional disability (assessed by the HAP(33-35)) and increased mortality. Data largely did not support the importance of HGST even if a link with some QoL domains has been found.

L. Li et al., 2008 was aimed to determine the validity of the $2 \mathrm{MWT}$ as a measure of exercise tolerance and physical performance in patients with cGVHD. Only the congress'poster of the study was available and it did not specify the age of the 51 patients involved. 
Adopted outcome measures consisted of: distance walked in 2 and 6 minutes; physical functioning; energy expenditure and physical fitness; cGVDH symptoms and global severity.

As a result, 2MWT showed strong positive correlation with 6MWT, SF-36(36)and HAP(37). Authors concluded that 2MWT and 6MWT (38)could be used interchangeably in patients with cGVHD.

\section{Discussion}

The paper summarized and critically evaluated rehabilitative assessment and treatment tools currently used in HSCT pediatric patients. Ten eligible studies were found: 3 regarding in-patient phase, 1 referring both to in-patient and out-patient phase, 2 about out-patients phase and 4 which connected to cGVHD. One review referring both to in-patient and out-patient phase has been selected too.

Referring to in-patients phase, 2/3 studies chose as outcomes measures QoL, functional motor abilities and fatigue. Fatigue was evaluated in all studies through the PedsQL Multidimensional Fatigue Scale(39) (Table1).

About intervention programs, 2/3 suggested 5 weekly sessions each with a duration of 50 minutes. Endurance and strength training were proposed by $3 / 3$ studies and aerobic activities by $2 / 3$ (Table2).

All articles regarding out-patients phase considered HRQOL as an outcome measures. 2/3 studies chose as other outcomes measures muscle resistance and strength, cardiovascular functioning and gas-exchange-data. Functional motor abilities and $\mathrm{VO}_{2 \text { peak }}$ were investigated in 2/3 and common tests ware used: respectively Time Up and Go (TUG), Time Up and Down Stairs Test (TUDS) $(28,29)$ and Treadmill Test (Table3).

About intervention programs, all studies tested programs with a frequency of 3 weekly session, in $2 / 3$ each session had a duration of 60 minutes. The three selected areas were the same chose for in-patient phase: $3 / 3$ studies proposed aerobic activities and endurance and strength exercises, $2 / 3$ stretching exercises. $2 / 3$ study utilized a logbook for patients in order to empower them and increase their participation to rehabilitation program (Table4).

All studies on cGVHD individuated as outcome variables physical functioning, 2/4 suggested SF-36 as evaluation instrument. 3/4 articles investigate cGVHD symptoms and cGVHD global severity, 2/3 used the Lee cGVHD Symptom Scale(40). Other outcomes measures investigated by 2/4 works were: 
ROM, mortality, cGVHD response and functional abilities. QoL, energy expenditure, physical fitness and strength were evaluated in 2/4 articles using the same tools, respectively: FACT-BMT(41), HAP and 2MWT (Table5).

Intervention program was referred only in 2 studies which agree on stretching exercises and occupational therapy, with particular attention for functional abilities, strength and resistance training(Table6).

\section{Conclusion}

In conclusion, rehabilitation programs improve physical morbidity in paediatric HSCT population and facilitates reintegration into everyday life $(18,24,25,27-29)$. However, today there is no agreement around a most suitable and effective exercise program. First of all, validated assessment instruments are needed in order to control outcome variables that are capable of indicating efficacy of intervention programs. They are both requested by therapists for everyday clinical practice than by scientific literature for clinical trials.

Regarding exercise interventions, pending further studies, our analysis can be a starting point for the formulation of intervention program: most investigated outcome measures were QoL and physical functioning and abilities. About rehabilitative intervention, there were heterogeneous data regarding duration of intervention and frequency of sessions, otherwise most studies suggested that each rehabilitative session has to last about of 60 minutes for 3-5 weekly/sessions. Most suggested exercises areas were: aerobic activities, strength training and endurance/resistance training accompanied by articular motion and stretching exercises. These matches with Physical Activity Guidelines for Americans (42) mentioned by Majhail et al., 2012(43) about management of muscle and soft tissue HSCT complications but it's clear that guidelines need to be adapted and compared with scientific literature about HSCT patients whose physical functioning isn't comparable with that of healthy subjects.

Further studies are strictly necessary to base rehabilitation practice of pediatric HSCT recipients on scientific evidence to ensure them a real reintegration into everyday life. 


\section{$\underline{\text { References }}$}

1. Perumbeti A, Ronald A Sacher, Emmanuel C Besa, Robert J Arceci, Timothy P Cripe, Daniel Efiom-Ekaha, et al. Hematopoietic Stem Cell Transplantation. Medscape Gen Med [Internet]. 2014 Mar 31 [cited 2015 Aug 30]; Available from: http://emedicine.medscape.com/article/208954-overview

2. Bresters D, van Gils ICM, Kollen WJW, Ball LM, Oostdijk W, van der Bom JG, et al. High burden of late effects after haematopoietic stem cell transplantation in childhood: a single-centre study. Bone Marrow Transplant. 2010 Jan;45(1):79-85.

3. Armenian SH, Sun C-L, Kawashima T, Arora M, Leisenring W, Sklar CA, et al. Long-term health-related outcomes in survivors of childhood cancer treated with HSCT versus conventional therapy: a report from the Bone Marrow Transplant Survivor Study (BMTSS) and Childhood Cancer Survivor Study (CCSS). Blood. 2011 Aug 4;118(5):1413-20.

4. Hovi L, Kurimo M, Taskinen M, Vettenranta J, Vettenranta K, Saarinen-Pihkala UM. Suboptimal long-term physical performance in children and young adults after pediatric alloSCT. Bone Marrow Transplant. 2010 Apr;45(4):738-45.

5. Ness KK, Bhatia S, Baker KS, Francisco L, Carter A, Forman SJ, et al. Performance limitations and participation restrictions among childhood cancer survivors treated with hematopoietic stem cell transplantation: the bone marrow transplant survivor study. Arch Pediatr Adolesc Med. 2005 Aug;159(8):706-13.

6. Barrera M, Atenafu E, Andrews GS, Saunders F. Factors Related to Changes in Cognitive, Educational and Visual Motor Integration in Children who Undergo Hematopoietic Stem Cell Transplant. J Pediatr Psychol. 2008 Jun 1;33(5):536-46.

7. Cool VA. Long-term neuropsychological risks in pediatric bone marrow transplant: what do we know? Bone Marrow Transplant. 1996 Dec;18 Suppl 3:S45-9.

8. Phipps S, Dunavant M, Srivastava DK, Bowman L, Mulhern RK. Cognitive and academic functioning in survivors of pediatric bone marrow transplantation. J Clin Oncol Off J Am Soc Clin Oncol. 2000 Mar;18(5):1004-11.

9. Poppelreuter M, Weis J, Mumm A, Orth HB, Bartsch HH. Rehabilitation of therapy-related cognitive deficits in patients after hematopoietic stem cell transplantation. Bone Marrow Transplant. 2008 Jan;41(1):79-90.

10. Duell T, van Lint MT, Ljungman P, Tichelli A, Socie G, Apperley JF, et al. Health and Functional Status of Long-Term Survivors of Bone Marrow Transplantation. Ann Intern Med. 1997 Feb 1;126(3):184-92. 
11. Taskinen MH, Kurimo M, Kanerva J, Hovi L. Physical performance of nontransplanted childhood ALL survivors is comparable to healthy controls. J Pediatr Hematol Oncol. 2013 May;35(4):276-80.

12. Barrera M, Boyd-Pringle LA, Sumbler K, Saunders F. Quality of life and behavioral adjustment after pediatric bone marrow transplantation. Bone Marrow Transplant. 2000 Aug;26(4):427-35.

13. Barrera M, Atenafu E, Hancock K. Longitudinal health-related quality of life outcomes and related factors after pediatric SCT. Bone Marrow Transplant. 2009 Aug;44(4):249-56.

14. Felder-Puig R, di Gallo A, Waldenmair M, Norden P, Winter A, Gadner H, et al. Health-related quality of life of pediatric patients receiving allogeneic stem cell or bone marrow transplantation: results of a longitudinal, multi-center study. Bone Marrow Transplant. 2006 Jul;38(2):119-26.

15. Lawitschka A, Güclü ED, Varni JW, Putz M, Wolff D, Pavletic S, et al. Health-related quality of life in pediatric patients after allogeneic SCT: development of the PedsQL Stem Cell Transplant module and results of a pilot study. Bone Marrow Transplant. 2014 Aug;49(8):10937.

16. Fiuza-Luces C, Garatachea N, Simpson RJ, Berger NA, Ramírez M, Lucia A. Understanding graft-versus-host disease. Preliminary findings regarding the effects of exercise in affected patients. Exerc Immunol Rev. 2015;21:80-112.

17. Fraser CJ, Bhatia S, Ness K, Carter A, Francisco L, Arora M, et al. Impact of chronic graftversus-host disease on the health status of hematopoietic cell transplantation survivors: a report from the Bone Marrow Transplant Survivor Study. Blood. 2006 Oct 15;108(8):2867-73.

18. Carenzio G, Gherardi P, Bardoni MT, Zecca M, Bonetti F, Locatelli F, et al. Rehabilitation of chronic graft versus host disease in children. A clinical series. Eur Medicophysica. 2007 Dec;43(4):445-50.

19. Currie DM, Ludvigsdottir GK, Diaz CA, Kamani N. Topical treatment of sclerodermoid chronic graft vs. host disease. Am J Phys Med Rehabil Assoc Acad Physiatr. 2002 Feb;81(2):143-9.

20. Beredjiklian PKMD, Drummond DSMD, Dormans JPMD, Davidson RSMD, Brock GTMD, August CMD. Orthopaedic Manifestations of Chronic Graft-Versus-Host Disease. J Pediatr Orthop. 1998 Oct;18(5):572-5.

21. Smith SR, Haig AJ, Couriel DR. Musculoskeletal, Neurologic, and Cardiopulmonary Aspects of Physical Rehabilitation in Patients with Chronic Graft-versus-Host Disease. Biol Blood Marrow Transplant J Am Soc Blood Marrow Transplant. 2014 Oct 23;

22. San Juan AF, Wolin K, Lucía A. Physical activity and pediatric cancer survivorship. Recent Results Cancer Res Fortschritte Krebsforsch Prog Dans Rech Sur Cancer. 2011;186:319-47. 
23. Huang T-T, Ness KK. Exercise interventions in children with cancer: a review. Int J Pediatr. 2011;2011:461512.

24. Chamorro-Viña C, Ruiz JR, Santana-Sosa E, González Vicent M, Madero L, Pérez M, et al. Exercise during hematopoietic stem cell transplant hospitalization in children. Med Sci Sports Exerc. 2010 Jun;42(6):1045-53.

25. Rosenhagen A, Bernhörster M, Vogt L, Weiss B, Senn A, Arndt S, et al. Implementation of structured physical activity in the pediatric stem cell transplantation. Klin Pädiatr. 2011 May;223(3):147-51.

26. Van Haren IEPM, Timmerman H, Potting CM, Blijlevens NMA, Staal JB, Nijhuis-van der Sanden MWG. Physical exercise for patients undergoing hematopoietic stem cell transplantation: systematic review and meta-analyses of randomized controlled trials. Phys Ther. 2013 Apr;93(4):514-28.

27. West SL, Gassas A, Schechter T, Egeler RM, Nathan PC, Wells GD. Exercise intolerance and the impact of physical activity in children treated with hematopoietic stem cell transplantation. Pediatr Exerc Sci. 2014 Aug;26(3):358-64.

28. Chamorro-Viña C, Guilcher GMT, Khan FM, Mazil K, Schulte F, Wurz A, et al. EXERCISE in pediatric autologous stem cell transplant patients: a randomized controlled trial protocol. BMC Cancer. 2012;12:401.

29. San Juan AF, Chamorro-Viña C, Moral S, Fernández del Valle M, Madero L, Ramírez M, et al. Benefits of intrahospital exercise training after pediatric bone marrow transplantation. Int $\mathrm{J}$ Sports Med. 2008 May;29(5):439-46.

30. Hayes S, Davies PSW, Parker T, Bashford J, Newman B. Quality of life changes following peripheral blood stem cell transplantation and participation in a mixed-type, moderate-intensity, exercise program. Bone Marrow Transplant. 2004 Mar;33(5):553-8.

31. Pidala J, Chai X, Martin P, Inamoto Y, Cutler C, Palmer J, et al. Hand grip strength and 2-minute walk test in chronic graft-versus-host disease assessment: analysis from the Chronic GVHD Consortium. Biol Blood Marrow Transplant J Am Soc Blood Marrow Transplant. 2013 Jun;19(6):967-72.

32. Li L, Chan L, Gerber LH Validation of 2-Minute Walk Test as a Measure of Exercise Tollerance and Physical Performance in Patients with Chronic Graft Versus Host Disease. Arch Phys Med and Rehabil. 2008; 89:e28.

33. Parsons S, Phipps S, Sung L, Baker K, Pulsipher M, Ness K. NCI, NHLBI/PBMTC First International Conference on Late Effects after Pediatric Hematopoietic Cell Transplantation: Health-Related Quality of Life, Functional, and Neurocognitive Outcomes. Biol Blood Marrow Transplant. 2012 Feb;18(2):162-71. 
34. Eames GM, Crosson J, Steinberger J, Steinbuch M, Krabill K, Bass J, et al. Cardiovascular function in children following bone marrow transplant: a cross-sectional study. Publ Online 01 January 1997 Doi101038sjbmt1700600. 1997 Jan 1;19(1):61-6.

35. Hogarty AN, Leahey A, Zhao H, Hogarty MD, Bunin N, Cnaan A, et al. Longitudinal evaluation of cardiopulmonary performance during exercise after bone marrow transplantation in children. J Pediatr. 2000 Mar;136(3):311-7.

36. McHorney CA, Ware JE, Raczek AE. The MOS 36-Item Short-Form Health Survey (SF-36): II. Psychometric and clinical tests of validity in measuring physical and mental health constructs. Med Care. 1993 Mar;31(3):247-63.

37. Herzberg PY, Heussner P, Mumm FHA, Horak M, Hilgendorf I, von Harsdorf S, et al. Validation of the human activity profile questionnaire in patients after allogeneic hematopoietic stem cell transplantation. Biol Blood Marrow Transplant J Am Soc Blood Marrow Transplant. 2010 Dec;16(12):1707-17.

38. ATS Committee on Proficiency Standards for Clinical Pulmonary Function Laboratories. ATS statement: guidelines for the six-minute walk test. Am J Respir Crit Care Med. 2002 Jul 1;166(1):111-7.

39. Varni JW, Burwinkle TM, Katz ER, Meeske K, Dickinson P. The PedsQL in pediatric cancer: reliability and validity of the Pediatric Quality of Life Inventory Generic Core Scales, Multidimensional Fatigue Scale, and Cancer Module. Cancer. 2002 Apr 1; 94(7):2090-106.

40. Lee S k, Cook EF, Soiffer R, Antin JH. Development and validation of a scale to measure symptoms of chronic graft-versus-host disease. Biol Blood Marrow Transplant J Am Soc Blood Marrow Transplant. 2002;8(8):444-52.

41. McQuellon RP, Russell GB, Cella DF, Craven BL, Brady M, Bonomi A, et al. Quality of life measurement in bone marrow transplantation: development of the Functional Assessment of Cancer Therapy-Bone Marrow Transplant (FACT-BMT) scale. Bone Marrow Transplant. 1997 Feb;19(4):357-68.

42. www.health.gov. 2008 Physical activity guidelines for Americans. US Department of Health and Human Services;

43. Majhail NS, Rizzo JD, Lee SJ, Aljurf M, Atsuta Y, Bonfim C, et al. Recommended Screening and Preventive Practices for Long-term Survivors after Hematopoietic Cell Transplantation. Bone Marrow Transplant. 2012 Mar;47(3):337-41. 
